\title{
Proceeding
}

Asia Pacific Conference on Performance Analysis of Sport, 21-24 April 2014. Langkawi, Malaysia

\section{Time-motion and heart-rate characteristics of adolescent female foil fencers}

\author{
MATTHEW JAMES WYLDE $\checkmark$, LOW CHEE YONG \\ Performance Enhancement Institute, Singapore Sports School, Singapore
}

\begin{abstract}
Wylde, M.J., \& Yong, L.C. (2015). Time-motion and heart-rate characteristics of adolescent female foil fencers. J. Hum. Sport Exerc., 9(Proc2), pp.S699-S706. Purpose: The aims of this study was to understand the time-motion and heart-rate characteristics of adolescent female foil fencing, explore the differences between the 5-touch and 15-touch bouts and compare these results with previous analysis of elite female foil fencers. Methods: Eight adolescent female foil fencers (age $14.3 \pm 1.2 \mathrm{y}$, stature $157 \pm 3.8 \mathrm{~cm}$, body mass $45.9 \pm 5.2 \mathrm{~kg}$ ) were analysed at a youth fencing competition. Each fencer wore a heart-rate monitor throughout the competition and bouts were filmed so time-motion analysis could be carried out. Five movement classifications were used for the time-motion analysis, this were grouped into three categories based on movement intensity: Low, Moderate and High. Results: High-intensity movement accounted for $8.8 \pm 4.4 \%$ of total bout time with a mean duration of $0.8 \pm 0.2 \mathrm{~s}$. A higher percentage of low-intensity movement was recorded in the 15-touch bouts, with a higher percentage of high-intensity movement recorded in the 5 -touch bouts. The mean duration of low-intensity movement was longer in the 15-touch bouts compared to the 5-touch bouts and a higher mean heart-rate was recorded in the 15-touch bouts compared to the 5-touch bouts. Conclusions: The results from this study suggest that adolescent female foil fencers perform a greater percentage of high-intensity movement in 5-touch bouts compared to 15-touch bouts. However, these fencers demonstrate a higher heart-rate in the 15-touch bouts. The practical application of this study is that adolescent female foil fencers must have a strong aerobic base to maintain a lower heart-rate in competition and ensure that fatigue does not become a limiting factor to performance. Key words: TIME-MOTION ANALYSIS, ADOLESCENT ATHLETES, FENCING.
\end{abstract}

\footnotetext{
Corresponding author. Performance Enhancement Institute, Singapore Sports School, Singapore 737913

E-mail: matthew_wylde@sportsschool.edu.sg

Asia Pacific Conference on Performance Analysis of Sport, 21-24 April 2014. Langkawi, Malaysia.

JOURNAL OF HUMAN SPORT \& EXERCISE ISSN 1988-5202

(c) Faculty of Education. University of Alicante

doi:10.14198/jhse.2015.10.Proc2.09
} 


\section{INTRODUCTION}

Fencing is an open-skill combat sport in which two athletes fight indirectly through their weapons. There are three distinct weapon categories (epee, foil and sabre) each with their own styles, techniques and areas for scoring. Fencing bouts are classified as either 5 -touch (first fencer to five points) or 15-touch (first fencer to 15 points). In competition, fencers initially compete in 5-touch bouts in a pool with 5 to 7 other fencers, the qualifiers from these pools go through to the direct elimination rounds of 15 -touches.

Until relatively recently there was a lack of scientific literature on the sport of fencing (Roi \& Bianchedi, 2008). However, in recent years there have been a number of papers published exploring a range of facets of fencing. One such paper explored the physiological characteristics of elite female epee fencers in simulated competition and found heart-rate responses at 86 to $90 \%$ of the fencers' maximum, high peak energy expenditures but low blood-lactate concentrations (Bottoms et al., 2011). This demonstrated that, in simulated competition, female epee fencers used primarily the anaerobic alactic energy system. The authors of this study suggested that further research, including time-motion analysis and evaluation of competitive scenarios, was required to better inform coaches on the physical demands being placed on elite fencers.

A subsequent study explored both these facets of elite female foil fencing (Wylde et al., 2013). This study found that high-intensity movements accounted for $6.2 \pm 2.5 \%$ of total bout time and that these highintensity movements had a mean duration of $0.7 \pm 0.1 \mathrm{~s}$. An overall work:recovery ratio of $1: 1.1$ was report with heart-rate responses at 90 to $95 \%$ of the fencers maximum. This study concluded that in addition to anaerobic alactic fitness, elite fencers must have a strong aerobic base to aid their recovery between bouts and ensure that fatigue does not inhibit their performance.

The studies in the current fencing literature have focused almost exclusively on the demands of senior elite fencing, meaning the demands of fencing in a youth development context remain relatively unexamined. Further research is necessary as it is not possible to generalise information gathered on elite athletes to youth athletes (Chiodo et al., 2012). The aims of this study are to understand the time-motion and heartrate characteristics of adolescent female foil fencing, explore the differences between the 5 -touch and 15touch bouts and compare these results with those found for elite female foil fencers (Wylde et al., 2013).

\section{MATERIAL AND METHODS}

Eight adolescent female foil fencers (age $14.3 \pm 1.2 \mathrm{y}$, stature $157 \pm 3.8 \mathrm{~cm}$, body mass $45.9 \pm 5.2 \mathrm{~kg}$ ) were analysed at the 2013 Singapore National Inter-School Fencing Championship. Each fencer wore a heart-rate monitor (Polar Electro Oy, Kempele, Finland) throughout the competition. Bouts involving these fencers were filmed from a balcony above the fencing hall and time-motion analysis was carried out using SportsCode Pro (Sportstec ${ }^{\mathrm{TM}}$ Limited, Warriewood, Australia). For the time-motion analysis five movement classifications were used that were grouped into three categories based on movement intensity: Low, Moderate and High (Wylde et al., 2013). 
Table 1. Movement classifications and operational definitions (Wylde et al., 2013)

\begin{tabular}{|c|c|c|}
\hline Classification & Intensity & Operational Definition \\
\hline Stationary & Low & $\begin{array}{l}\text { Fencer is in a stationary position. } \\
\text { - Standing between engagements. } \\
\text { - In the "on guard" position }\end{array}$ \\
\hline Walking & Low & $\begin{array}{l}\text { Movement around the strip } \\
\text { - Between engagements. } \\
\text { - Walking away from the opponent as time is running } \\
\text { out during engagements. }\end{array}$ \\
\hline Engaged & Moderate & $\begin{array}{l}\text { Movement within the strip during engagement, prior to } \\
\text { an attacking / defensive movement. }\end{array}$ \\
\hline $\begin{array}{l}\text { Attack / Defence } \\
\text { Engagement }\end{array}$ & High & $\begin{array}{l}\text { Sustained attacking or defensive movement; } \\
\text { - Advancing up the stripe. } \\
\text { - Retreating down the stripe. }\end{array}$ \\
\hline $\begin{array}{l}\text { Attack / Defence } \\
\text { Burst }\end{array}$ & High & $\begin{array}{l}\text { Explosive attacking or defensive movements: } \\
\text { - A lunge where at some point both feet leaves the } \\
\text { ground. } \\
\text { - An attack where the fencers charges at the } \\
\text { opponent. } \\
\text { - A leap / jump back to avoid an opponents' attack. } \\
\text { - A sudden downward movement to duck under an } \\
\text { opponents' attack. }\end{array}$ \\
\hline
\end{tabular}

The differences between playing positions were analysed using magnitude-based Cohen's (1988) effect size (ES) with modified qualitative descriptors (Hopkins, 2000). Effect sizes were assessed using these criteria: $<0.20=$ trivial, 0.20 to $0.60=$ small, $>0.60$ to $1.20=$ moderate, $>1.20$ to $2.00=$ large, and $>2.00=$ very large. Ninety per cent confidence limits $( \pm 90 \% \mathrm{CL})$ were calculated to indicate the precision of the estimate of observed effects.

\section{RESULTS}

Across all bouts, high-intensity movement accounted for $8.8 \pm 4.4 \%$ of total bout time. There were "large" effect sizes observed between the High category and the other two categories. 


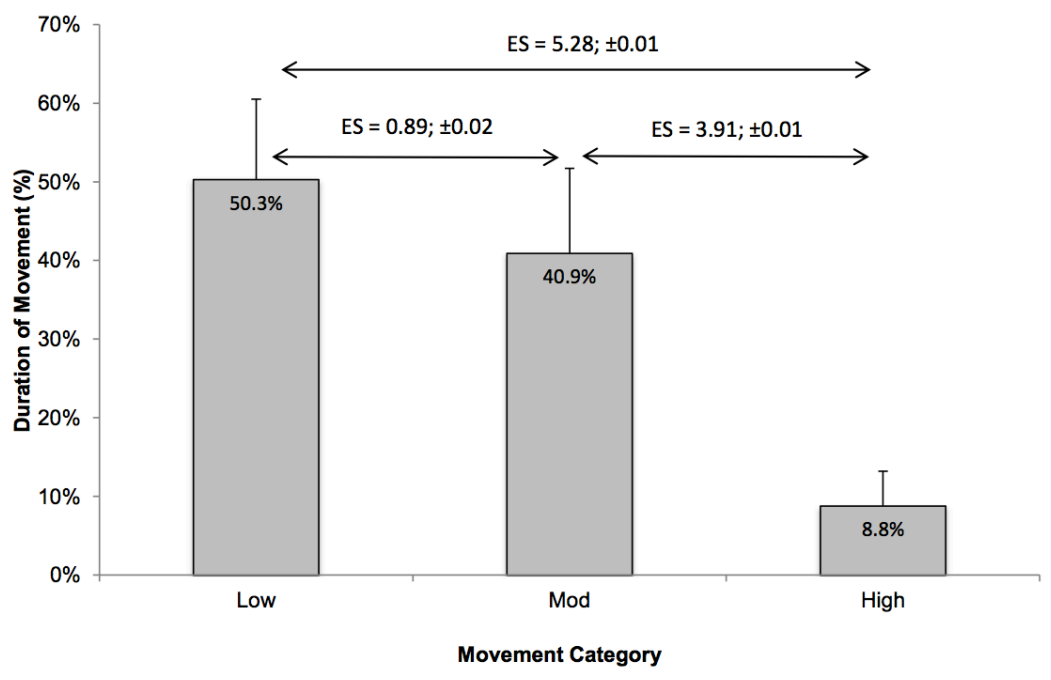

Figure 1. Percentage breakdown of movement categories, mean \pm SD.

The high-intensity movements had a mean duration of $0.8 \pm 0.2 \mathrm{~s}$. Again there were "large" effect sizes observed between the High category and the other two categories.

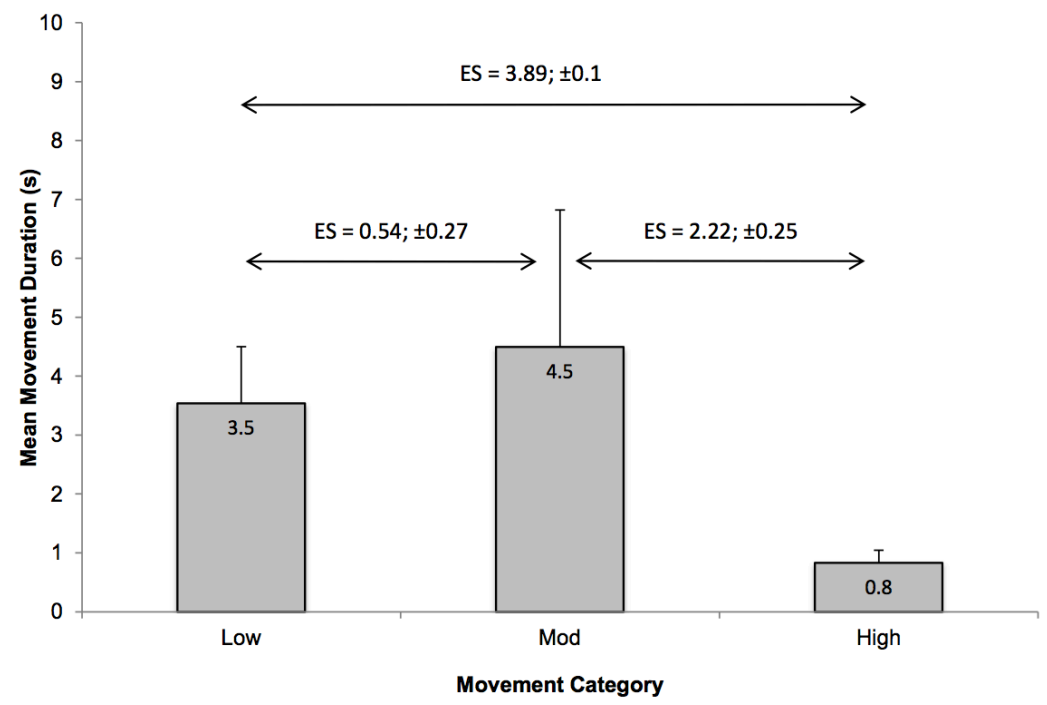

Figure 2. Mean duration of movements, mean \pm SD.

A higher percentage of low-intensity movement was recorded in the 15-touch bouts, with a higher percentage of high-intensity movement recorded in the 5 -touch bouts. In both cases "moderate" effect sizes were recorded. 


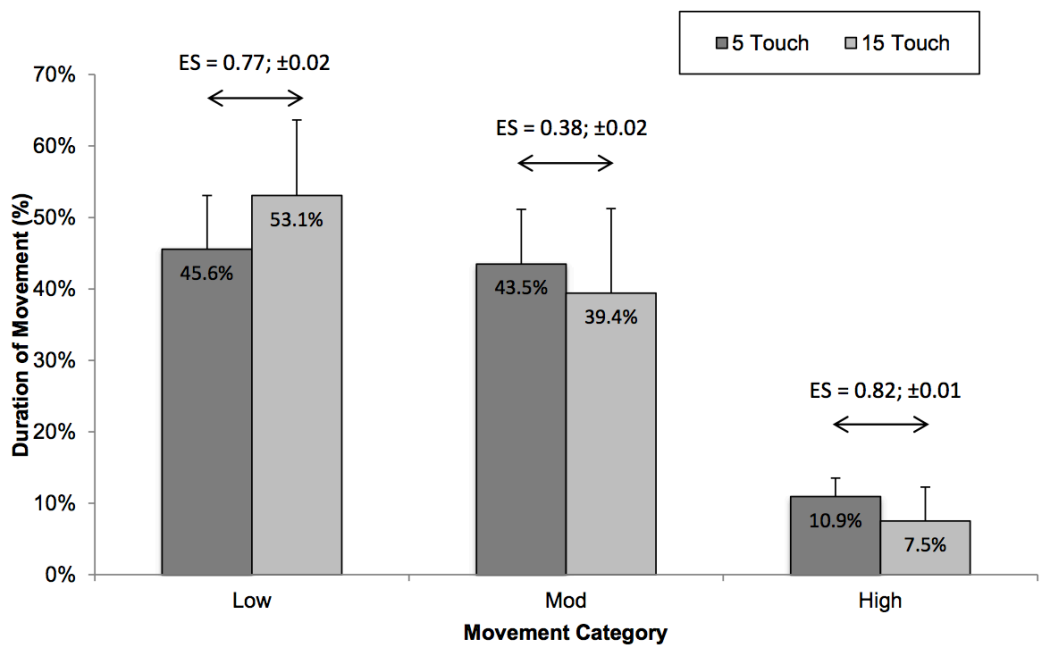

Figure 3. Percentage breakdown of movement categories by bout types, mean \pm SD

The mean duration of low-intensity movement was longer in the 15 -touch bouts compared to the 5 -touch bouts, with a "moderate" effect size being recorded.

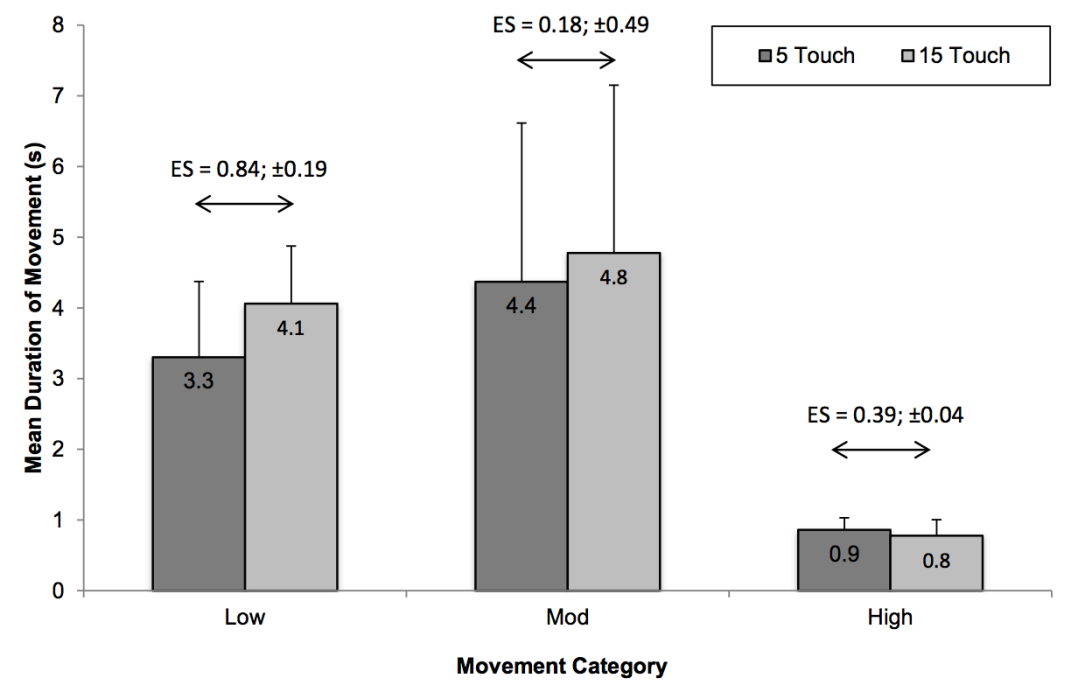

Figure 4. Mean duration of movements by categories by bout types, mean \pm SD

A higher mean heart-rate was recorded in the 15 -touch bouts compared to the 5-touch bouts, with a "moderate" effect size being recorded. 


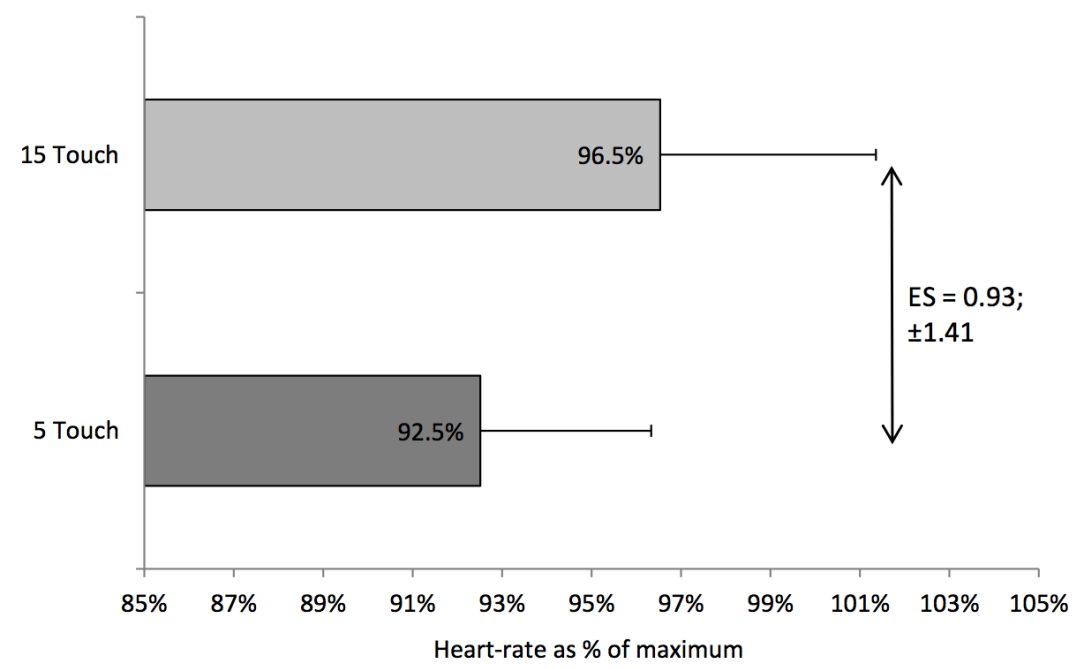

Figure 5. Mean heart-rate as a percentage of the fencers' maximum recorded in a multi-stage 20-m shuttle run to exhaustion test

\section{DISCUSSION}

Adolescent female foil fencers are required to perform a greater percentage of high-intensity movement in 5 -touch bouts than in 15-touch bouts. However, the fencers are required to maintain a higher heart-rate in the longer 15-touch bouts. This may be indicative of the higher level of competitiveness in the direct elimination rounds compared to the pool rounds. As the fencers in the direct elimination round will be of a similar standard each point will be keenly contested, meaning the fencers will need to perform more preparatory work for each point. This would account for the lower percentage of high-intensity movement in the 15 -touch as well as the increased heart-rate.

The results from this study are generally comparable with the study of elite female foil fencing (Wylde et al., 2013), with a work:recovery ratio of 1:1 for the adolescent fencers compared to 1:1.1 for the elite fencers. The adolescent fencers performed a greater percentage of high-intensity activity compared to the senior fencers, with a "moderate" effect size between the two groups. The senior fencers demonstrated a longer duration of low-intensity activity compared to the adolescent fencers, with a "moderate" effect size reported. This suggests that the elite fencers maximise the breaks in play to aid their physical recovery between points, helping to prevent fatigue. This is supported by the heart-rate data, with the adolescent fencers reaching heart-rates closer to their maximum compared to the elite group.

The heart-rate data for the elite and adolescent foil fencers were both higher than those reported in the study of simulated epee competition (Bottoms et al., 2011). This suggests that higher adrenaline associated with the stress of actual competition is a key factor in increasing heart-rate in fencing. This is supported by a study of taekwondo that found increases in heart-rate and adrenaline in actual competition compared to an exercise protocol that simulated the demands of competition (Bridge et al., 2013). Unpublished data from a pilot study of adolescent fencers conducted at the Singapore Sports School found that levels of Salivary Alpha Amylase $(\mathrm{sAA})$, a biomarker for stress, were significantly higher $(p<0.05)$ when taken directly after simulated fencing bouts than when taken before or 30 mins after the bouts (Low \& Choong, 
2012). These results are similar to those found in young taekwondo athletes in official competition (Capranica et al., 2012). These results support that notion that the elevated heart-rate demonstrated by adolescent fencers is partly a result of competition anxiety.

\section{Practical Applications}

While the work recovery ratios recorded for adolescent and elite female foil fencers were nearly exactly the same, the adolescent fencers demonstrated a higher heart-rate response. This highlights the importance of building a strong aerobic base in adolescent fencers to limit fatigue by maintaining a lower heart-rate during competition. As with elite fencing, aerobic endurance training should be integrated into training, through bouts, lessons and endurance-oriented footwork (Weichenberger et al., 2012).

As competition anxiety appears to be a contributing factor to the elevated heart-rate in adolescent female foil fencers, a strategy may be required to help young fencers cope with the stresses of competition. For example, mental skills training could be utilised to enable adolescent fencers to lower their heart-rate and control adrenaline levels during competition.

\section{CONCLUSIONS}

The results from this study suggest that adolescent female foil fencers perform a greater percentage of high-intensity movement in 5-touch bouts compared to 15-touch bouts. However, these fencers demonstrate a higher heart-rate in the 15-touch bouts. Overall these results are comparable with the study of elite female foil fencers (Wylde et al., 2013), with similar work:recovery ratios recorded in the two studies. It was found that adolescent fencers perform a greater amount of high-intensity movement while elite fencers perform longer durations of low-intensity movement. The maximum heart-rates for the adolescent foil fencers were higher than those of the elite foil fencers and the elite epee fencers (Bottoms et al., 2011).

These results suggest that adolescent female foil fencers must have a strong aerobic base to maintain a lower heart-rate in competition and ensure that fatigue does not become a limiting factor to performance. Competition anxiety may also be a cause of the elevated heart-rate so programmes should be put in place to help adolescent fencers deal in the stresses of competition.

\section{REFERENCES}

1. Bottoms, L.M., Sinclair, J., Gabrysz, T., Szmantlan-Gabrysz, U., \& Price, M.J. (2011). Physiological responses and energy expenditure to simulated epee fencing in elite fencers. Serb J Sport Sci, $5(1)$, pp.17-20.

2. Bridge, C.A., McNaughton, L.R., Close, G.L., \& Drust, B. (2013). Taekwondo exercise protocols do not recreate the physiological responses of championship combat. Int J Sport Med, 34(7), pp.573582.

3. Capranica, L., Lupo, C., Cortis, C., Chiodo, S., Cibelli, G., \& Tessitore, A. (2012). Salivary cortisol and alpha-amylase reactivity to taekwondo competition in children. Eur J Appl Physiol, 112(2), pp.647-653.

4. Chiodo, S., Tessitore, A., Lupo, C., Ammendolia, A., Cortis, C., \& Capranica, L. (2012). Effects of official youth taekwondo competitions on jump and strength performance. Eur J Sport Sci, 12(2), pp.113-120.

5. Cohen, J. (1988). Statistical Power Analysis for the Behavioral Sciences (2). New Jersey: Lawrence Erlbaum. 
6. Hopkins, W.G. (2002). A scale of magnitudes for effect statistics. Available at: sportsci.org/resource/stats/index.html.

7. Low, C.Y., \& Choong, G.C.W. (2012). Salivary alpha amylase reactivity to fencing in adolescents (Unpublished Pilot Study).

8. Roi, G.S., \& Bianchedi, D. (2008). The science of fencing, implications for performance and injury prevention. Sport Med, 38(6), pp.465-481.

9. Weichenberger, M., Liu, Y., \& Steinacker, J.M. (2012). A test for determining endurance capacity in fencers. Int J Sport Med, 33, pp.48-52.

10. Wylde, M.J., Tan, F.H.Y., \& O'Donoghue, P.G. (2013). A time-motion analysis of elite women's fencing. Int J Perform Anal, 13(2), pp.365-376. 